Bull. Austral. Math. Soc.

$30 \mathrm{~F} 10,30 \mathrm{~F} 30,30 \mathrm{~F} 35$

Vol. 43 (1991) [399-405]

\title{
ON HOLOMORPHIC DIFFERENTIALS OF SOME ALGEBRAIC FUNCTION FIELD OF ONE VARIABLE OVER C
}

\author{
Ja KYUNG Koo
}

We give holomorphic differentials of some algebraic function field $K$ of complex dimension one which is a generalisation of a hyperelliptic field.

\section{INTRODUCTION}

Let $K$ be an algebraic function field of one variable over $C$. Then $K$ is generated over $\mathbf{C}$ by two generic points $x$ and $y$ of a plane curve defined by some equation $f(X, Y)=0$, if $f$ is the irreducible polynomial vanishing on $(x, y)$. Here $x$ is transcendental over $\mathbf{C}$ and $y$ is algebraic over the rational function field $F=\mathbf{C}(\boldsymbol{x})$. In the sense of the theory of Riemann surfaces one can identify $K$ with the field of meromorphic functions on a compact Riemann surface $([1,6,7,8])$. Thus all the statements parallel one another.

Let $\Omega$ be the set of differentials ( = differential one forms) of $K$. Then $\Omega$ is a one dimensional vector space over $K([3,5,8])$, which allows us to express it as $\Omega=K \cdot d x$. If $w=y d x$ is a differential of $K$ and $\mathfrak{P}$ a prime divisor of $K$, we define the order of $w$ at $\mathfrak{P}$ by $v_{\mathfrak{P}}(w)=\nu_{\mathfrak{P}}(y d x / d t)$ where $t$ is a local uniformising parameter in $K_{\mathfrak{P}}$ (the completion of $K$ with respect to the $\mathfrak{P}$-adic topology). As is well known, $K_{\mathfrak{P}}$ is isomorphic to the field $\mathbf{C}((t))$ of formal power series and hence $\nu_{\mathfrak{P}}$ is the standard valuation on $C((t))$. Note that $v_{\mathfrak{P}}(w)$ is independent of the choice of $t$ and $v \mathfrak{p}(w)=0$ for almost all prime divisors $\mathfrak{P}$. We call $w \in \Omega$ a holomorphic differential (or a differential of the first kind) if $v_{\mathfrak{P}}(w) \geqslant 0$ for all $\mathfrak{P}$. If $\Omega_{1}$ is the set of holomorphic differentials and $g$ is the genus of $K$, then $\Omega_{1}$ is a $g$-dimensional vector space over $C([1,2,3,6,8])$. Therefore we can describe all the holomorphic differentials when we are given an algebraic function field $K$.

In this paper we find holomorphic differentials of $K=\mathbf{C}(x, y)$ for which $x$ and $y$ satisfy the following equation :

$$
y^{n}=A\left(x-a_{1}\right)^{n_{1}}\left(x-a_{2}\right)^{n_{2}} \cdots\left(x-a_{\ell}\right)^{n_{\ell}}
$$

where all $a_{i}(\in \mathbf{C})$ are distinct, $A \in \mathbf{C}^{\times}, n \geqslant 2, n_{j} \geqslant 1(j=1,2, \ldots, \ell)$ and $\left(n, n_{1}, \ldots, n_{\ell}\right)=1$. Observe that $\left(n, n_{1}, \ldots, n_{\ell}\right)$ is the greatest common divisor of

Received 6th June, 1990.

Copyright Clearance Centre, Inc. Serial-fee code: 0004-9729/91 SA2.00+0.00. 
the integers $n, n_{1}, \ldots, n_{\ell}$. In particular, $K$ is called a hyperelliptic field provided that $n=2, \ell \geqslant 5$ and $n_{1}=\cdots=n_{\ell}=1$, in which case one can give an explicit basis $\left\{w_{1}, \ldots, w_{g}\right\}$ for $\Omega_{1}$ as follows $([2,3,8])$ :

$$
w_{j}=\frac{x^{j-1} d x}{y}, \quad 1 \leqslant j \leqslant g=\left[\frac{\ell-1}{2}\right]
$$

where $y=\sqrt{A} \sqrt{\left(x-a_{1}\right) \ldots\left(x-a_{\ell}\right)}$ and $[r]$ denotes the largest integer $\leqslant r$.

\section{Preliminaries}

To start with we need to show that the polynomial

$$
Y^{n}-A\left(X-a_{1}\right)^{n_{1}} \ldots\left(X-a_{\ell}\right)^{n_{\ell}}
$$

is irreducible over $\mathbf{C}$.

Lemma 1. Let $D$ be a unique factorisation domain of characteristic $O$ and $F$ be the quotient field of $D$. For $a \in D$, put $a=\pi_{1}^{e_{1}} \pi_{2}^{e_{3}} \cdots \pi_{l}^{e_{l}}$ with $\pi_{j}$ prime elements in $D$. If $n$ is a positive integer such that $\left(n, e_{1}, \ldots, e_{\ell}\right)=1$, then the polynomial $\varphi(Y)=Y^{n}-a$ over $D$ is irreducible over $F$.

ProOF: Let $\xi$ be a primitive $n$-th root of unity in $F$ and $\alpha$ be an element of the algebraic closure $\bar{F}$ of $F$ for which $\varphi(\alpha)=0$. Since $F(\alpha)$ is a (finite) Galois extension of $F$, let $G$ be the Galois group of $F(\alpha)$ over $F$ and $m=[F(\alpha): F]$. It suffices to show that $m=n$.

Suppose $m<n$. Take an $F$-automorphism $\sigma$ from $G$. Then

$$
\sigma(\alpha)^{n}=\sigma\left(\alpha^{n}\right)=\sigma(a)=a ;
$$

hence $\sigma(\alpha)=\xi^{\nu(\sigma)} \alpha$ for $\nu(\sigma) \in \mathbf{Z} / n \mathbf{Z}$. We derive from this an injective homomorphism $\nu$ from $G$ into the additive group $\mathrm{Z} / n \mathrm{Z}$. Thus we may view $G$ as a subgroup of $\mathrm{Z} / n \mathrm{Z}$ which is cyclic of order $n$. Let $G=\langle\tau\rangle$. Since $F(\alpha)$ is Galois over $F,|G|=m$ divides $n$. We observe by the definition of $\nu$ that $\tau(\alpha)=\xi^{\nu(\tau)} \alpha$ implies $\tau^{m}(\alpha)=\xi^{\nu\left(\tau^{m}\right)} \alpha=$ $\xi^{m \nu(r)} \alpha$.

$$
\text { But, } \tau^{m}(\alpha)=\alpha ; \text { hence }
$$

$$
m \nu(\tau) \equiv 0 \quad(\bmod n)
$$

On the other hand, by (2),

and

$$
\begin{aligned}
& (\tau(\alpha))^{m}=\xi^{m \nu(r)} \alpha^{m}=\alpha^{m} \\
& (\tau(\alpha))^{m}=\tau\left(\alpha^{m}\right) .
\end{aligned}
$$


Therefore $\alpha^{m}$ belong to $F$. Set $\alpha^{m}=b$. Then $b^{n / m}=\left(\alpha^{m}\right)^{n / m}=\alpha^{n}=a$.

Since $m$ divides $n$, let $b^{n / m}=b^{r}$. Obviously $r>1$. Now we have $a=b^{r}$ with $a=\pi_{1}^{e_{1}} \cdots \pi_{\ell}^{e_{\ell}}$, from which we conclude that $r$ is a common divisor of $e_{1}, e_{2}, \ldots$, and $e_{\ell}$. Since $r$ also divides $n,\left(n, e_{1}, \ldots, e_{\ell}\right)>1$. This is a contradiction.

Lemma 1 asserts that the polynomial in (*) is irreducible over $\mathbf{C}$.

ThEOREM 2 (Riemann-Hurwitz's formula). Let $K$ be an algebraic function field of one variable over $\mathbf{C}$ of genus $g$, and $F=\mathbf{C}(x)$ for $x \in K-\mathbf{C}$ such that $[K: F]=n$. Let $\mathfrak{P}$ be a prime divisor of $K$ and $e_{\mathfrak{P}}$ be the ramification index of $\mathfrak{P}$. Then

$$
\frac{1}{2} \sum_{\mathfrak{P}}\left(e_{\mathfrak{P}}-1\right)=n+g-1 .
$$

Proof: $([2,3,4,5,8])$.

Since $\mathfrak{P}$ lies over some prime divisor $\mathfrak{F}_{\alpha}$ of $F(\alpha \in \widehat{\mathbf{C}}$ the extended complex plane), we have the following diagram :

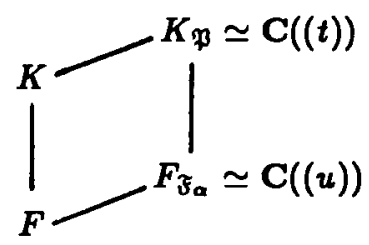

where $F_{\mathfrak{F}_{\alpha}}$ is the completion of $F$ and $u$ is a local uniformising parameter in $F_{\mathfrak{F}_{\alpha}}$. If $e=e\left(\mathfrak{P} \mid \mathfrak{F}_{\alpha}\right)$ is the ramification index of $\mathfrak{P}$ over $\mathfrak{F}_{\alpha}$, then $u=t^{e}$. Hence

$$
u= \begin{cases}x-\alpha & \text { if } \alpha \neq \infty \\ 1 / x & \text { if } \alpha=\infty\end{cases}
$$

implies that

$$
v_{\mathfrak{P}}(d x)=\nu_{\mathfrak{P}}\left(\frac{d x}{d t}\right)= \begin{cases}e-1 & \alpha \neq \infty \\ -e-1 & \alpha=\infty\end{cases}
$$

\section{MaIN Theorem}

Following the Riemann-Hurwitz's formula one can derive the genus $g$ of $K$ as follows

$$
g=\frac{1}{2} n(\ell-1)-\frac{1}{2}\left\{\sum_{j=1}^{\ell}\left(n, n_{j}\right)+(n, N)\right\}+1
$$


where $N=\sum_{j=1}^{\ell} n_{j}$. We first consider the differential of the form $w=d x / y$ with $y=\sqrt[n]{A} \sqrt[n]{\left(x-a_{1}\right)^{n_{1}} \cdots \cdots\left(x-a_{\ell}\right)^{n_{\ell}}}$.

It follows from (3) that

$$
v_{\mathfrak{P}}(d x)= \begin{cases}1-1=0 & \text { if } \alpha \neq \infty \text { and } a_{j} \\ \frac{n}{\left(n, n_{j}\right)}-1 & \text { if } \alpha=a_{j} \\ -\frac{n}{(n, N)}-1 & \text { if } \alpha=\infty\end{cases}
$$

Next, we obtain from (1) that

$$
n \nu \mathfrak{P}(y)=\nu \mathfrak{P}\left(\left(x-a_{1}\right)^{n_{1}}\right)+\cdots+\nu \mathfrak{p}\left(\left(x-a_{\ell}\right)^{n_{\ell}}\right)
$$

because $v_{\mathfrak{P}}(y)=\nu \mathfrak{P}(y)$ and $\nu_{\mathfrak{P}}(A)=0$. Since all $\left(x-a_{j}\right)$ belong to the field $F$,

$$
n \nu_{\mathfrak{P}}(y)=n_{1} e_{\mathfrak{P}} \nu_{\mathfrak{F}_{\alpha}}\left(\left(x-a_{1}\right)\right)+\cdots+n_{\ell} e_{\mathfrak{P}} \nu_{\mathfrak{F}_{\alpha}}\left(\left(x-a_{\ell}\right)\right)
$$

where $\nu_{\mathcal{F}_{\alpha}}$ is the discrete (exponential) valuation of $F$. Thus, by (6), (7)

$$
\nu_{\mathfrak{P}}(y)= \begin{cases}\frac{1}{n}\left\{n_{1} \cdot 1 \cdot 0+\cdots+n_{\ell} \cdot 1 \cdot 0\right\}=0, & \alpha \neq \infty \text { and } a_{j} ; \\ \frac{1}{n}\left\{0+\cdots+0+n_{j} \cdot \frac{n}{\left(n, n_{j}\right)} \cdot 1+0+\cdots+0\right\}=\frac{n_{j}}{\left(n, n_{j}\right)}, & \alpha=a_{j} ; \\ \frac{1}{n}\left\{n_{1} \cdot \frac{n}{(n, N)} \cdot-1+\cdots+n_{\ell} \cdot \frac{n}{(n, N)} \cdot-1\right\}=-\frac{N}{(n, N)}, & \alpha=\infty .\end{cases}
$$

Since $v_{\mathfrak{P}}(w)=v_{\mathfrak{P}}(d x)-\nu_{\mathfrak{P}}(y)$, it follows from (5) and (7) that for a prime divisor $\mathfrak{P}$ over $\mathfrak{F}_{\boldsymbol{\alpha}}$

$$
\nu_{\mathfrak{P}}(w)= \begin{cases}0 & \text { if } \alpha \neq \infty \text { and } a_{j} \\ \frac{n-\left(n_{j}+\left(n, n_{j}\right)\right)}{\left(n, n_{j}\right)} & \text { if } \alpha=a_{j} \\ \frac{N-(n+(n, N))}{(n, N)} & \text { if } \alpha=\infty\end{cases}
$$

Therefore, $d x / y$ is holomorphic if and only if $n \geqslant n_{j}+\left(n, n_{j}\right)(1 \leqslant j \leqslant \ell)$ and $N \geqslant n+(n, N)$. We readily see from this that the necessary conditions are

$$
n>n_{j} \quad(j=1,2, \ldots, \ell) \text { and } N>n .
$$

For instance, look at the case $n=3$ and $\ell=3$. Then by (4)

$$
g=3-\frac{1}{2}\left\{\left(3, n_{1}\right)+\left(3, n_{2}\right)+\left(3, n_{3}\right)+\left(3, n_{1}+n_{2}+n_{3}\right)\right\}+1,
$$


and hence, by the restriction (8), we come up with the following table :

\begin{tabular}{|c|c|c|c|c|}
\hline$n_{1}$ & $n_{2}$ & $n_{3}$ & validity & $g$ \\
\hline 1 & 1 & 1 & $\times$ & 1 \\
\hline 1 & 1 & 2 & 0 & 2 \\
\hline 1 & 2 & 2 & 0 & 2 \\
\hline 2 & 2 & 2 & 0 & 1 \\
\hline
\end{tabular}

In the first case $d x / y$ is not a holomorphic differential; then what is the basis element of $\Omega_{1}$ ? In the other cases $d x / y \in \Omega_{1}$, moreover in the last case every holomorphic differential is a constant multiple of $d x / y$. But, there still remains a question in the second and the third case; what is another basis element $w$ independent of $d x / y$ ?

To have complete answers we consider the differentials of the form

$$
w=\frac{\prod_{j=1}^{\ell}\left(x-a_{j}\right)^{k_{j}} d x}{y^{m}}
$$

where $m \geqslant 1, k_{j} \geqslant 0$ and $y=\sqrt[n]{A} \sqrt[n]{\left(x-a_{1}\right)^{n_{1}} \cdots \cdots\left(x-a_{\ell}\right)^{n_{\ell}}}$.

Theorem 3. A differential $w$ in (9) is holomorphic if and only if $n\left(k_{j}+1\right) \geqslant$ $m n_{j}+\left(n, n_{j}\right)(1 \leqslant j \leqslant \ell)$ and $m N \geqslant\left(\sum_{j=1}^{\ell} k_{j}+1\right) n+(n, N)$.

Proof: For a prime divisor $\mathfrak{P}$ over $\mathfrak{F}_{\boldsymbol{\alpha}}$,

$$
\begin{aligned}
v_{\mathfrak{P}}(w) & =v_{\mathfrak{P}}(d x)+\sum_{j=1}^{\ell} k_{j} \nu_{\mathfrak{P}}\left(\left(x-a_{j}\right)\right)-m \nu_{\mathfrak{P}}(y) \\
& =v_{\mathfrak{P}}(d x)+\sum_{j=1}^{\ell} k_{j} e_{\mathfrak{P}} \nu_{\mathfrak{F}_{\alpha}}\left(\left(x-a_{j}\right)\right)-m \nu_{\mathfrak{P}}(y) .
\end{aligned}
$$

Here

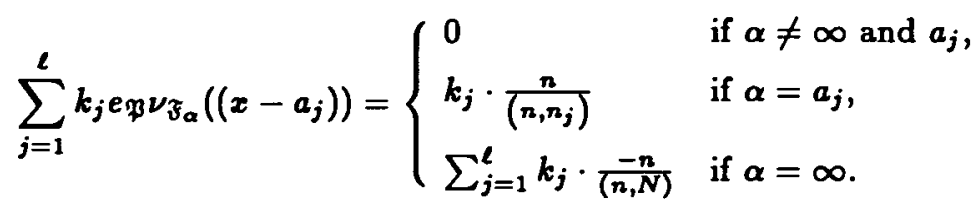

Therefore, by (5), (7) and (10)

$$
v_{\mathfrak{P}}(w)= \begin{cases}0, & \alpha \neq \infty \text { and } a_{j}, \\ \frac{n\left(k_{j}+1\right)-\left(m n_{j}+\left(n, n_{j}\right)\right)}{\left(n, n_{j}\right)}, & \alpha=a_{j}, \\ \frac{m N-\left(\left(\sum_{j=1}^{\ell} k_{j}+1\right) n+(n, N)\right)}{(n, N)}, & \alpha=\infty,\end{cases}
$$

from which we have the theorem. 


\section{Applications}

In Section 3 we see that $d x / y$ is not a holomorphic differential when $n=3$ and $n_{1}=n_{2}=n_{s}=1$. Applying Theorem 3, however, we can get appropriate basis element to this case. Since $N=n_{1}+n_{2}+n_{3}=3,\left(n, n_{j}\right)=1$ and $(n, N)=3, w$ in (9) is holomorphic if and only if $3\left(k_{1}+1\right) \geqslant m+1,3\left(k_{2}+1\right) \geqslant m+1,3\left(k_{3}+1\right) \geqslant m+1$ and $3 m \geqslant 3\left(k_{1}+k_{2}+k_{3}+1\right)+3$, or equivalently $3 k_{1} \geqslant m-2,3 k_{2} \geqslant m-2,3 k_{3} \geqslant m-2$ and $m \geqslant k_{1}+k_{2}+k_{3}+2(m>1)$.

Taking smallest possible integers, $m=2$ and $k_{1}=k_{2}=k_{3}=0$. Therefore every holomorphic differential in this case is a constant multiple of $d x / y^{2}$ with $y=$ $\sqrt[3]{A} \sqrt[3]{\left(x-a_{1}\right)\left(x-a_{2}\right)\left(x-a_{3}\right)}$

Likewise, in the second and third cases holomorphic bases are

and

$$
\begin{aligned}
& \left\{\frac{d x}{y}, \frac{\left(x-a_{3}\right) d x}{y^{2}}\right\} \quad\left(y=\sqrt[3]{A} \sqrt[3]{\left(x-a_{1}\right)\left(x-a_{2}\right)\left(x-a_{3}\right)^{2}}\right) \\
& \left\{\frac{d x}{y}, \frac{\left(x-a_{2}\right)\left(x-a_{3}\right) d x}{y^{2}}\right\} \quad\left(y=\sqrt[3]{A} \sqrt[3]{\left(x-a_{1}\right)\left(x-a_{2}\right)^{2}\left(x-a_{3}\right)^{2}}\right)
\end{aligned}
$$

respectively.

In the case of a hyperelliptic field $K$, we can derive a different basis for $\Omega_{1}$ from the one described in Section 1 such as $\left\{w_{1}^{\prime}, \ldots, w_{g}^{\prime}\right\}$ with

$$
w_{k}^{\prime}=\frac{\prod_{j=1}^{\ell}\left(x-a_{j}\right)^{k-1} d x}{y^{2 k-1}} \quad(1 \leqslant k \leqslant g)
$$

and

$$
y=\sqrt{A} \sqrt{\left(x-a_{1}\right) \cdots\left(x-a_{\ell}\right)}
$$

\section{REFERENCES}

[1] C. Chevalley, Introduction to the theory of algebraic functions of one variable, AMS Mathemstical Surveys No. 6, 1951.

[2] H.M. Farkas and I. Kra, Riemann surfaces (Springer-Verlag, Heidelberg, New York, Berlin, 1980).

[3] O. Foster, Lectures on Riemann surfaces (Springer-Verlag, Berlin, Heidelberg, New York, 1981).

[4] R. Hartshorne, Algebraic geometry (Springer-Verlag, Berlin, Heidelberg, New York, 1977).

[5] S. Lang, Introduction to algebraic and abelian functions (Springer-Verlag, Berlin, Heidelberg, New York, 1982).

[6] C.L. Siegel, Topics in complex function theory Vol I, $I I$ (Wiley-Interscience, 1970). 
[7] G. Shimura, Introduction to the arithmetic theory of automorphic functions (Princeton University Press, 1971).

[8] G. Springer, Introduction to Riemann surface (Chelsea Publishing Co., New York, 1957).

Korea Advanced Institute of Science and Technology

Department of Mathematics

Taejon 305-701

Korea 